\title{
Conventional and Advanced Diagnostic Tools in Oral Cancer with Emphasis on Role of Dentist and Early Detection
}

\author{
${ }^{1}$ Smit Singla, ${ }^{2}$ Akhilesh Verma, ${ }^{3}$ Snehil Goyal, ${ }^{4}$ Itika Singla, ${ }^{5}$ Anjali Shetty
}

\begin{abstract}
The prevalence of oral cancer is increasing worldwide day by day. Those who suffer from oral cancer have low survival rate compared with other cancer, mainly due to delay in the diagnosis, metastases, and presence of secondary tumors. For the dental profession, it is really challenging to diagnose oral precancer or oral cancer, particularly in its early stage of the disease. Early screening and advanced diagnostic tools play an important role in the early diagnosis and treatment planning of patients suffering from oral cancer. The diagnosis method usually includes histopathology and visualization adjuncts, such as toluidine blue, vizilite, etc. This study reviews the development of new upcoming tools for early detection, emphasis on the importance of role of dentist, and early detection of oral cancer.
\end{abstract}

Keywords: Early detection, Oral cancer, Screening.

How to cite this article: Singla S, Verma A, Goyal S, Singla I, Shetty A. Conventional and Advanced Diagnostic Tools in Oral Cancer with Emphasis on Role of Dentist and Early Detection. J Postgrad Med Edu Res 2017;51(3):128-133.

Source of support: Nil

Conflict of interest: None

\section{INTRODUCTION}

Worldwide, oral cancer accounts for 2 to $4 \%$ of all cancer diagnoses. Around 400,000 new cases of oral and pharynx cancer are reported every year. Depending upon the geographical region, prevalence of oral cancer varies as it represents approximately $10 \%$ of all cancer in Pakistan, whereas it accounts for approximately $45 \%$ in the Indian

\footnotetext{
${ }^{1}$ Reader, ${ }^{2}$ Senior Resident, ${ }^{3,4}$ General Practitioner ${ }^{5}$ Assistant Professor

${ }^{1}$ Department of Oral Medicine and Radiology, Daswani Dental College and Research Centre, Kota, Rajasthan, India

${ }^{2}$ Department of Dentistry, Mathura Das Mathur Hospital Jodhpur, Rajasthan, India

${ }^{3,4}$ Department of Dentistry, Guru Kirpa Dental Clinic \& Implant Centre, Bhadaur, Punjab, India

${ }^{5}$ Department of Oral Medicine and Radiology, A.J. Institute of Dental Sciences, Mangaluru, Karnataka, India

Corresponding Author: Smit Singla, Reader, Department of Oral Medicine and Radiology, Daswani Dental College and Research Centre, Kota, Rajasthan, India, Phone: +919915029271, e-mail: smitcool2@yahoo.co.in
}

subcontinent as a whole. Anatomic sites of oral cancer also vary with the geographic location..$^{1-3}$ Anatomic sites vary as the use of tobacco and related habits varies with geographic location. With change in geographic location, the habit also varies, which most importantly includes the percentage of tobacco used by the subjects.

Males are more commonly affected compared with females, with a ratio of 1.5:1. Older age group is the mostly affected age group, but now the trend is changing as the incidence rate of affected individuals less than 45 years of age has doubled from 1973. It may be due to change in lifestyle. Often, patients report to dentists or other specialists during the late stage of the disease; this leads to 45 and 32\% 5-year survival rate after treatment of stages III and IV respectively. Although treatment for oral cancer is widely available, the mortality rate of overall disease is still high, i.e., $49 \%{ }^{4-6}$

\section{ROLE OF DENTIST}

Oral cavity and perioral region are most frequently examined by the dentist. So, dentists have an important role in detecting oral signs and symptoms of any disorder. Dentists even have an important role for the treatment and follow-up of any oral mucosal lesions. Dental examination should be done thoroughly. There should be primary goal to diagnose the lesion properly even in the early stage of the disorder as signs and symptoms of most of the diseases mimic each other. Diagnosis should be done before the metastases progress. After proper diagnosis, the dentist should decide whether abnormality requires further investigation. Diagnostic delay is an important factor that should be prevented by offering required investigation as prompt care of patient with oral cancer reduces the morbidity and mortality and offers a favorable prognosis of the disease to increase the quality of life. In scientific literature, a delay in dental treatment can be classified as (1) patients' delay, the period between the patient's first symptom and the first appointment with a health professional regarding the symptom described by the patient; and (2) service/professional delay, which corresponds to the period between the patient's first appointment with a health professional and definite diagnosis. Several studies indicate that dentists do not 
have sufficient knowledge regarding the etiology and diagnosis. Dentists should perform screening examination on every patient, especially in smokers and alcohol users every year regardless of their age. ${ }^{1,7-9}$

\section{IMPORTANCE OF EARLY DETECTION}

The importance lies in the early detection in premalignant or early stage of the disease so that we can potentially limit the need for most invasive and disfiguring levels of the treatment to improve the quality of life of the patient. Early treatment means less invasive treatment that can prevent altered eating and swallowing patterns, salivary gland dysfunction, loss of structures, such as teeth, bone, and soft tissues including tongue, etc. In 2014 , Singla et $\mathrm{a}^{10}$ concluded that at 60 Gy of radiation therapy, the unstimulated salivary flow rate becomes zero, which leads to dysgeusia, xerostomia, and oral mucositis. Early diagnosis reduces morbidity, improves the patient's long-term survival, and increases the quality of life. Sankaranarayanan et $\mathrm{al}^{11}$ conducted a study in which they concluded there was reduction of mortality in smokers and drinkers of 43 and $22 \%$ respectively, after conducting oral cancer screening program.

\section{CLASSIFICATION}

\section{Oral Examination}

Oral cavity should be examined for oncological variations, which is simple, noninvasive, and within the skills of all dentists. Practitioner performs the examination usually with incandescent light, gauze, a mouth mirror, and magnification. There is a wide range of alterations seen in the oral cavity of patients suffering from oral precancer and cancer disorders. Alteration includes color change, i.e., red, white, or mixed; textural change, i.e., erosion, keratosis, granularity, loss of elasticity, fissuring or mixed; and additional signs include ulceration, induration, bone invasion, tooth mobility, and pain. In the later course of disease, lymphatic infiltration occurs. ${ }^{4,12}$

Oral examination is not able to discriminate between the progressive, malignant, and those nonprogressive counterparts. Malignant changes may occur without any clinical changes in the oral cavity. In some studies, sensitivity and specificity vary between 58 and $94 \%$ and 76 and $98 \%$ respectively. So, other diagnostic tools should be used along with clinical oral examination to detect early changes. ${ }^{13,14}$

\section{Vital Staining}

\section{Toluidine Blue}

It is a vital dye that has been used in the past for the detection of mucosal abnormalities of the cervix and the oral cavity. It is an acidophilic metachromatic dye, which is used to stain acidic tissue component, sulfate, carboxylate, and phosphate radicals, such as deoxyribonucleic acid (DNA) and ribonucleic acid (RNA), but not normal mucosa. As in malignancy, DNA synthesis increased and tumor cells are randomly arranged that helps in rapid dye penetration, which is the proposed mechanism of toluidine blue staining. First, the patient is asked to rinse with $1 \%$ acetic acid for 20 seconds followed by rinse with water twice for 20 seconds; after that the patient is asked to rinse the mouth with 5 to $10 \mathrm{cc}$. $1 \%$ toluidine blue solution followed by rinsing with $1 \%$ acetic acid solution (5 oz.) for about 1 minute again followed by water rinse. Dark blue stain is considered as positive for malignancy and light blue stain is considered positive for premalignant lesion unless proved by biopsy. Noninvasive, simple, inexpensive indication area for biopsy are the advantages whereas taste, stain remaining for 4 to 6 hours, and false-positive results are the disadvantages of toluidine blue. ${ }^{15,16}$ Overall, the sensitivity of toluidine blue staining for the detection of oral carcinomas varies between 78 and $100 \%$ and the specificity varies between 31 and $100 \%{ }^{17}$

\section{Lugol's lodine}

Lugol's iodine is formed by $4 \mathrm{gm}$ of potassium iodide and $2 \mathrm{gm}$ of iodine in $100 \mathrm{cc}$ of distilled water. First, the patient is asked to rinse with $1 \%$ acetic acid for 20 seconds, followed by water rinse for 20 seconds. Later, Lugol's iodine solution is applied to the suspicious lesion with cotton bud for 20 to 30 seconds and examined for retention of stain. Brown stain is considered as positive, while without retention it is considered as negative. Basis of this stain is glycogen content as normal epithelium has more glycogen content compared with malignant lesion. So, Lugol's iodine stains normal mucosa. Lugol's iodine staining even helps to know about differentiation of the lesions as poorly differentiated lesions without glycogen content do not have retention of Lugol's solution as compared with mildly differentiated lesions. Double staining technique which means use of Lugol's iodine along with toluidine chloride helps in differentiating the inflammatory lesion. Double staining technique is very useful to determine the biopsy site in suspicious cases. $^{15,18,19}$

\section{Methylene Blue}

It is a dark-green color odorless powder at room temperature, which generates blue solution when dissolved in water. Recently, it is suggested that methylene blue induces selective cancer cell apoptosis. It is cheaper and has low toxicity compared with toluidine blue, so can be used for oral screening on a large scale. ${ }^{20,21}$ 


\section{Rose Bengal Dye}

Rose Bengal has been used to detect nonhealthy epithelial mucosa. Du et al ${ }^{22}$ conducted a study in which six lesions with normal clinical appearance were stained by Rose Bengal staining. So, the authors concluded that it can be used as a diagnostic procedure, but further research is required.

\section{Cytopathological Examination}

\section{Oral Exfoliative Cytology}

In this, a collecting device may be swab, spatula, or brush and is placed and rotated against the mucosal surface, and cells are collected. These cells should be spread on a glass slide to prepare a smear. These slides were examined by pathologist after fixation and staining. It can detect epithelial dysplasia, carcinoma, and atypical results. Navone et $\mathrm{al}^{23}$ found this technique quite good for examination.

\section{Micronucleus Assay with Acridine Orange}

In this technique, alcohol-fixed smear is taken to distilled water, then smear is rinsed with water. After that, smear is rinsed in $1 \%$ acetic acid for few seconds, followed by two changes of distilled water for about 1 minute. Then, the smear will be stained with diluted acridine orange solution at $\mathrm{pH} 6.0$ for 3 minutes, and staining smear will be rinsed with buffer at $\mathrm{pH} 6$ for about 1 minute. After that, smear was differentiated with $0.1 \mathrm{M}$ calcium chloride solution for $1 / 2$ to 1 minute, then washed in phosphate buffer and mounted in the same. Frequency of micronuclei will be evaluated under fluorescent microscope. Singla et $\mathrm{al}^{24}$ concluded that micronucleus is a noninvasive diagnostic tool to determine microscopic changes even during the normal clinical appearance.

\section{Oral Brush Biopsy}

Oral CDx is an oral biopsy system which is computerassisted that collects transepithelial cellular samples. In this procedure, a special brush is placed on the lesion and rotated for 10 to 15 times till it produces reddening or hemorrhagic spots. Cell material which is obtained through brush is placed on dry slide, fixed, and sent for analysis. In the first published study in the United States, the brush biopsy reportedly detected correctly all cases of carcinoma. ${ }^{15,25,26}$

\section{Liquid-based Cytology}

Liquid-based cytology (LBC) is introduced to eliminate many disadvantages of conventional exfoliate cytology. In LBC, sample is immersed in a tube containing preservative fluid, which fixes cells immediately. The fluid is centrifuged and makes high cellular confined zone on glass slide. ${ }^{27}$

\section{Tumor Markers and Biomarkers}

These markers may be produced by cancer cells or by the host in response to cancerous agents. For detecting oral malignant lesions, cytokeratin markers can be used as these have altered expression, especially of buccal mucosa of oral cavity. ${ }^{19}$

\section{Light-based Detection System}

\section{Chemiluminescence}

It means emission of light by chemical reaction. Vizilite is most commonly used under chemiluminescence, which emits bluish-white light in a wavelength of 430 to $580 \mathrm{~nm}$. In this technique, subject is asked to rinse with $1 \%$ acetic acid, which is done to remove the debris and to increase the visibility of nuclei due to mild cellular dehydration. The normal mucosa absorbs the light and appears blue, whereas abnormal mucosa appears aceto-white with brighter, sharper, more distant margins as it reflects the light. The reported sensitivity is $100 \%$ and specificity ranges from 0 to $14.2 \% .{ }^{15,28}$ Recently, Vizilite system was modified to be used along with toluidine blue. So, MicroLux DL was introduced.

\section{Tissue Fluorescence Imaging}

In this technique oral mucosa is illuminated with intense blue light with a wavelength range from 400 to $460 \mathrm{~nm}$. Normal mucosa appears pale green compared with abnormal mucosa, which appears darker due to altered structure and metabolism of epithelium and subepithelial stroma. Microlux, Orascoptic, and Veloscope are included in tissue fluorescence imaging. Sensitivity ranges from 97 to $98 \%$ and specificity ranges from 94 to $100 \% .{ }^{15,17}$

\section{Tissue Fluorescence Spectroscopy}

In this technique, various excitation of wavelength produced by small optical fiber and a spectrograph is used to analyze oral mucosa. This technique has high sensitivity and specificity for the detection of malignant lesion from healthy mucosa. As optical fiber is small, only small mucosal area is scanned and therefore, not suitable for large mucosal lesion. . $^{15,17}$

\section{Identafi 3000}

In this technique, anatomical imaging is used along with fluorescence, fiber optics, and confocal microscopy. As it is small in size, it is easily accessible in the unaccessible areas of the oral cavity. Besides detection, it is also used to examine tissue, i.e., detecting changes in angiogenesis with green amber light. Sensitivity of $82 \%$ and specificity of $87 \%$ are achieved to distinguish between neoplastic and nonneoplastic conditions of the oral cavity. ${ }^{29}$ 


\section{Optical Coherence Tomography}

Optical coherence tomography is based on interferometer. In this, it is possible to get excellent spatial resolution and real-time imaging by using low-coherence broadband near infrared light source. It is a noninvasive, nonradiative diagnostic tool. In dysplastic conditions, cell size, shape, nucleus size, nucleus arrangement are more randomly distributed compared with normal mucosa. So, light scattering becomes stronger and more fluctuated. Cross-sectional images can be obtained of any mucosal area, whether it is normal or abnormal without biopsy. By using surface plasmon resonant gold nanoparticle, contrast images can be obtained. ${ }^{15,30}$

\section{Fluorescence Photography}

Oral cancer, particularly oral squamous cell carcinoma, can be detected by fluorescence photography which is a noninvasive, rapid, simple, and reproducible method. Enlargement and progression of disease can be predicted by positivity of fluorescence. ${ }^{19,31}$

\section{Other Light-based Techniques}

Contact endoscopy and endoscopic high-frequency ultrasound have been used in oral cavity. These new imaging techniques yield promising results in oral cavity, with sensitivity $91.3 \%$ and specificity $100 \%{ }^{32}$ Narrow band spectrum optical filters used to enhance visualization of mucosal pattern are used in narrow band imaging. ${ }^{33}$ Further research is necessary to understand the diagnostic value of these technologies.

\section{Saliva-based Diagnostic}

Saliva can be used as diagnostic tool and it is an attractive, effective, noninvasive screening tool for the detection of oral cancer. It is based on the fact that salivary composition is altered in patients with oral cancer. Salivary diagnosis depends upon the quantitative analysis of specific salivary macromolecules, enzymes, cytokines, growth factors, metalloproteases, endothelin, telomerase, cytokeratines, messenger RNA, and DNA transcripts. Total sugar, protein-bound sialic acid, free sialic acid, sodium, calcium, immunoglobulin G, albumin, lactate dehydrogenase levels are much higher in saliva of oral cancer patients as compared with normal patients. Oxidative stress-related salivary parameters, epithelial tumor markers cytokeratin-19 fragments in serum (CYFRA 21-1), tissue polypeptide-specific antigen, RNA transcripts [insulin-like growth factor, matrix metalloproteinase (MMP)-2, MMP-9, interleukin-8 and 1B] levels are significantly altered in oral cancer patients. Studies have shown that saliva of oral cancer patients contains statherin, histatin 3, proline-rich proteins, spermidine N1 acetyltransferase, $\beta$-actin, and glyceraldehyde3-phosphate dehydrogenase. Levels of salivary soluble CD44 are significantly increased in oral cancer patients, but there is no correlation found between the level of CD44 and stage of the tumor. More studies are required to investigate further as, to date, saliva-based testing has not been incorporated into a commercial product. Presently, it is more costly and not easily accessible as it is done in some higher specific labs only. ${ }^{16,34}$

\section{Microfluidics}

It is also referred as lab-on-a-chip or micro-total-analysis systems. Microfluidics accept saliva and are suitable for handling living cells. Dysplastic cells can be detected in this chip. Stage and type of the cancer from which patient is suffering can be detected by this technology. It is suitable for use in clinics or operating room. ${ }^{30}$

\section{Deoxyribonucleic Acid Ploidy}

The nuclear DNA content is measured by DNA ploidy. If chromosomes become detached or are not uniformly distributed to the daughter cell, then chromosomal segregation is termed as unbalanced during mitosis. This is called aneuploidy and observed in dysplastic lesions. In this technique, cytological samples are compared with a reference group after staining and computer-assisted analysis is done for genomic instability. It is a noninvasive procedure with high specificity. ${ }^{15,35}$

\section{Biopsy}

For diagnosis, incisional and punch biopsies are more commonly used. Histopathological examination determines the nature of dysplasia, i.e., mild, moderate, or severe, as it is the gold standard for the diagnosis of potentially malignant disorders. For the pathologist, adequate sample size is required to determine the proper diagnosis. If the sample is not adequate, then there are more chances for discrepancy in the report.

\section{Imaging Modalities}

\section{Diffuse Reflectance Imaging}

When light enters the tissue it may be elastically scattered, inelastically scattered, or absorbed. The property of light depends on the tissue morphology, such as nuclear size, distribution, epithelial thickness, collagen content, amount of oxy and deoxy hemoglobin present in the exposed tissue. All these content varies during transformation from normal tissue to malignant one. The resulting signal of the light after interaction with tissue is detected by fibers and fed into a spectrometer interfaced 
with a computer. Diffuse reflectance spectroscopy is a noninvasive optical tool for early detection of malignant changes in the tissue of the oral mucosa. ${ }^{16,30}$

\section{Fluorescence Endoscopy}

Fluorescence endoscopy is much more accurate and specific than white light endoscopy. It involves the use of photosensitizing drug to visualize lesion through tissue fluorescence. Most commonly used photosensitizing drugs used in endoscopy are 5-aminolevulinic acid and hypericin. Malignant lesions have higher uptake of drug compared with normal mucosa. ${ }^{30}$

\section{Positron Emission Tomography Scan}

Early diagnosis and staging of the tumor can be done by positron emission tomography. Fluorodeoxyglucose is injected and scan is done. Even early involvement of lymph node can be detected. So, early diagnosis of oral cancer can be done by this technique.

\section{Others}

\section{Polymerase Chain Reaction-based Diagnostic Tools}

The polymerase chain reaction can be used in the study of cancer and provide the information on the genetic etiology of neoplasia, as it can detect mutations in oncogenes and tumor suppressor genes. There is difficulty in the interpretation of the results due to contamination, which is one of the major disadvantages other than being costeffective, selectivity, availability, etc. ${ }^{19,31}$

\section{CONCLUSION}

Potentially malignant disorders pose an important threat to the overall survival of an individual. The ability to identify lesions and to predict which lesions will undergo malignant transformation would facilitate early diagnosis and subsequent disease management tailored to the individual patient. The ultimate goals are to reduce both mortality and morbidity, and to improve patients' quality of life. Detection tools are becoming increasingly accurate and less invasive as studies continue to be published in order to determine the sensitivity and specificity of each detection mechanism. Integration of the adjuncts and tests discussed here can help uncover hidden lesions before they have the chance to progress into malignancy. Hence, all practitioners should be aware of the latest advancements and should try to incorporate them whenever feasible.

\section{REFERENCES}

1. Boyle P, Levin B, editors. World cancer report 2008. Lyon: IARC Press; 2008.
2. Siddiqui IA, Farooq MU, Siddiqui RA, Rafi SMT. Role of toluidine blue in early detection of oral cancer. Pak J Med Sci 2006 Apr-Jun;22(2):184-187.

3. Oral Cancer Facts. Available from: http://www.oralcancerfoundation.org/facts/index.htm. Accessed November 2015.

4. Agar NJM, Patel RS. Early detection, causes and screening of oral cancer. JSM Dent 2014;2(3):1039.

5. Lingen MW, Kalmar JR, Karrison T, Speight PM. Critical evaluation of diagnostic aids for the detection of oral cancer. Oral Oncol 2008 Jan;44(1):10-22.

6. Warnakulasuriya S. Global epidemiology of oral and oropharyngeal cancer. Oral Oncol 2009 Apr-May;45(4-5):309-316.

7. Tripathi N, Pathak S. Early detection of oral cancer: duties of a dental surgeon. J Adv Clin Res Insights 2015;2:44-45.

8. Alfano MC, Horowitz AM. Professional and community efforts to prevent morbidity and mortality from oral cancer. J Am Dent Assoc 2001 Nov;132 (Suppl):24S-29S.

9. Souza LM, Michel-Crosato E, Biazevic MGH, Antunes JLF. Scheduling delay in suspected cases of oral cancer. Rev Bras Epidemiol 2011 Dec;14(4):642-650.

10. Singla S, Naik V, Kini R, Shetty A. Cumulative dose related adverse effects of radiation therapy on oral cavity. Int J Med Appl Sci 2014;3(2):106-110.

11. Sankaranarayanan R, Ramdas K, Thomas G, Muwonge R, Thara S, Mathew B. Effect of screening on oral cancer mortality in Kerala, India: a cluster-randomised controlled trial. Lancet 2005 Jun;365(9475):1927-1933.

12. Sciubba JJ. Oral cancer and its detection. J Am Dent Assoc 2001 Nov;132:12S-18S.

13. Downer MC, Moles DR, Palmer S, Speight PM. A systematic review of test performance in screening for oral cancer and precancer. Oral Oncol 2004 Mar;40(3):264-273.

14. Kujan O, Glenny AM, Solan P. Screening for oral cancer. Lancet 2005;366:1265-1266.

15. Hasan S, Elongovan S. Conventional and advanced diagnostic aids in oral cancer screening - the journey so far. Int J Pharm Pharm Sci 2015;7(1):29-33.

16. Scully C, Bagan JV, Hopper C, Epstein JB. Oral cancer: current and future diagnostic techniques. Am J Dent 2008 Aug;21(4):199-209.

17. Lingen MW, Kalmar JR, Karrison T, Speight PM. Critical evaluation of diagnostic aids for the detection of oral cancer. Oral Oncol 2008 Jan;44(1):10-22.

18. Nagaraju K, Prasad S, Ashok L. Diagnostic efficiency of toluidine blue with Lugol's iodine in oral premalignant and malignant lesions. Indian J Dent Res 2010 Apr-Jun;2:218-223.

19. Masthan KMK, Babu NA, Dash KC, Elumala M. Advanced diagnostic aids in oral cancer. Asian Pac J Cancer Prev 2012;13(8):3573-3576.

20. Gilman PK. Methylene blue is a potent monoamine oxidase inhibitor. Can J Anaesth 2008 May;55(5):311-312.

21. Reddy GS, Rao KE, Kumar KK, Sekhar PC,Prakash Chandra KL, Ramana Reddy BV. Diagnosis of oral cancer: the past and present. J Orofac Sci 2014;6:10-16.

22. Du GF, Li CZ, Chen HZ, Chen XM, Xiao Q, Cao ZG, Shang SH, Cai X. Rose Bengal staining in detection of oral precancerous and malignant lesions with colorimetric evaluation: a pilot study. Int J Cancer 2007 May;120(9):1958-1963.

23. Navone R,Marsico A, RealeI,Pich A, Broccoletti R, PenteneroM, Gandolfo S. Usefulness of oral exfoliative cytology for the diagnosis of oral squamous dysplasia and carcinoma. Minerva Stomatol. 2004 Mar;53(3):77-86. 
24. Singla S, Naik V, Kini R, Shetty A. Micronucleus assay an early diagnostic tool to assess genotoxic changes in tobacco and related habits. Beheshti Univ Dent J 2014;32(3): 139-146.

25. Sciubba JJ. Improving detection of precancerous and cancerous oral lesions. Computer-assisted analysis of the oral brush biopsy. U.S. Collaborative OralCDx Study Group. J Am Dent Assoc 1999 Oct;130(10):1445-1457.

26. Bench MK. Oral brush biopsies. J Am Dent Assoc 2006 Mar;137(3):294.

27. Hayama FH, Motta ACF, Silva APG, Migliari DA. Liquidbased preparations versus conventional cytology: specimen adequacy and diagnostic agreement in oral lesions. Med Oral Patol Oral Cir Bucal 2005 Mar-Apr;10(2):115-122.

28. Oh ES, Laskin DM. Efficacy of the Vizilite system in the identification of oral lesions. J Oral Maxillofac Surg 2007 Mar;65(3):424-426.

29. Messadi DV. Diagnostic aids for detection of oral precancerous conditions. Int J Oral Sci 2013 Jun;5:59-65.
30. Nigam P, Prasad K, Tak J, Gupta V, Sinha A, Bali R, Grewal P. Advanced Diagnostic Aids in Early Detection of Oral Cancer. J Adv Med Dent Scie Res 2014;2(3):39-43.

31. Satoskar S, Dinakar A. Diagnostic aids in early oral cancer detection - a review. JIAOMR 2006;18:82-89.

32. Mallia RJ, Thomas SS, Mathews A, Kumar R. Laser induced autofluorescence spectral ratio reference standard for early discrimination of oral cancer. Cancer 2008;112(7):1503-1512.

33. Piazza C, Cocco D, Del Bon F, Mangili S, Nicolai P, Majorana A, Bolzoni Villaret A, Peretti G. Narrow band imaging and high definition television in evaluation of oral and oropharyngeal squamous cell cancer: a prospective study. Oral Oncol 2010 Apr;46(4):307-310.

34. Naghipur S. A review of diagnostic aids for oral cancer screenings in general practice dentistry. Int Dent J Student's Res 2013;2(2):10-16.

35. Bradley G, Odell EW, Raphael S, Ho J, Le LW, Benchimol S, Kamel-Reid S. Abnormal DNA content in oral epithelial dysplasia is associated with increased risk of progression to carcinoma. Br J Cancer 2010 Oct;103(9):1432-1442. 